\title{
A Study of the Characteristics of the White Matter in Schizophrenic Patients with Defective or Non- defective Symptoms by Diffuse Tensor Imaging
}

\section{Wen-jie Shi}

Department of psychiatry Huai'an No.3 People's Hospital

\section{Tai-peng Sun}

Department of psychology Huai'an No.3 People's Hospital

\section{Li-rong Zhuang}

Department of psychiatry Huai'an No.3 People's Hospital

\section{Hua Feng}

Department of psychology Huai'an No.3 People's Hospital

\section{Peng Yan}

Department of psychiatry Huai'an No.3 People's Hospital

\section{Cong-jie Wang}

Department of psychiatry Huai'an No.3 People's Hospital

\section{Zheng Wang}

Department of Radiology Huai'an No.3 People's Hospital

Cheng-bing Huang ( $D$ 896687526@qq.com )

Department of Radiology Huai'an No.3 People's Hospital

\section{Research article}

Keywords: Deficit schizophrenia, Diffusion tensor imaging (DTI), Tract-based spatial statistics (TBSS)

Posted Date: May 19th, 2020

DOI: https://doi.org/10.21203/rs.3.rs-26837/v1

License: (1) (1) This work is licensed under a Creative Commons Attribution 4.0 International License. Read Full License 


\section{Abstract}

\section{Background}

Deficit schizophrenia (DS) is a set of highly homogenous schizophrenia subtypes characterized by primary and persistent negative symptoms. Previous research studies have found that the negative symptoms of schizophrenia are closely related to the impairment of brain structure and function. This study seeks to explore the characteristics of white matter in schizophrenic patients with defective or nondefective symptoms by diffuse tensor imaging (DTI).

\section{Methods}

According to the defective schizophrenia diagnostic criteria and ICD-10 diagnostic criteria, 30 patients with DS and 30 patients with non-defective schizophrenia (NDS) were enrolled into the research study. DTI imaging data of the white matter were collected by $1.5 \mathrm{~T}$ magnetic resonance imaging scanner. Then a tract-based spatial statistics (TBSS) method was used to compare the fractional anisotropy (FA) values of the white matter fiber between the two groups.

\section{Results}

The TBSS analysis results showed that the FA values in the right side of the knee of the corpus callosum (MNI:14,36,-7), right anterior radio-coronal region (MIN:11,34,3) and the right hippocampal region (MIN:30,34,16) in the DS patients were significantly lower compared with those of the NDS patients (all $p<0.05)$. The FA values in the right side of the knee of the corpus callosum was significantly correlated with the time from onset to treatment $(r=-0.350, p<0.001)$, PANSS-negative symptom score $(r=-0.157$, $p=0.007)$. The FA values in the right anterior radio-crown region was positively correlated with PANSSnegative symptom score $(\mathrm{r}=0.306, p=0.048)$. The right hippocampus was negatively correlated with years of education ( $r=-0.614, p=0.020)$, duration of antipsychotics using $(r=-0.140, p=0.022)$, and PANSSnegative symptom score $(r=-0.637, p=0.040)$.

\section{Conclusions}

In schizophrenic patients with defective symptoms, the structural integrity of white matter fibers was more seriously damaged in the three brain regions including the right knee of the corpus callosum, the right anterior region of the right radiative crown, and the right hippocampus. These white matter lesions are closely related to patient characteristics such as years of education, duration from onset to treatment, duration of anti-psychotic, and severity of negative symptoms.

\section{Background}

Deficit schizophrenia (DS) is a set of highly homogenous schizophrenia subtypes characterized by primary and persistent negative symptoms ${ }^{[1]}$. In contrast to non-deficit schizophrenia (NDS), the cognitive impairment of patients with DS is more significant, and the response to drug therapy, brain stimulation 
therapy, psychotherapy, and other treatment options are not ideal ${ }^{[2]}$. Previous research studies have found that the negative symptoms of schizophrenia are closely related to the impairment of brain structure and function ${ }^{[3]}$. It is reasonable to predict that there are also significant differences between DS and NDS in the impairment of brain structure and function; however, this has not been reported in the extant medical literature.

As a non-traumatic imaging method, diffusion tensor imaging (DTI) is widely used in the study of structural changes that occur in the brains of patients with schizophrenia. Compared with healthy people, patients with schizophrenia have decreased fractional anisotropy (FA). Some researchers have interpreted this as white matter degeneration in schizophrenia and reported the association between negative symptoms and reduced FA values in multiple brain regions (such as the frontal and temporal regions) ${ }^{[4,5]}$. In consideration of the differences between the clinical characteristics of DS and NDS, it is reasonable to assume that the changes in the white matter may also be different. However, there is no consistent result in this field.It would be meaningful to to explore the differences between DS and NDS in clinical characteristics for clinical diagnosis and treatment guidance.Therefore,The purpose of our study is to compare the changes of white matter between DS and NDS patients by DTI.

\section{Methods}

\section{Study population}

Case group: The out-patients or in-patients with schizophrenia were recruited from the Department of

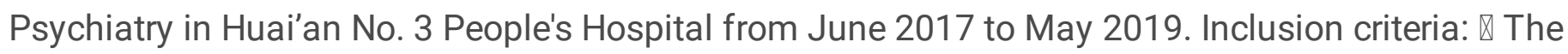
diagnosis meets the criteria for schizophrenia based on the International Classification of Diseases-10 (ICD-10); $₫$ The DS meets the diagnostic criteria of the Chinese version of the schedule for the deficit syndrome $(\mathrm{SDS})^{[6]}$; $\otimes$ The patient's age is between 18 and 60 years. Exclusion criteria: $\otimes$ Pregnant or lactating women; $\otimes$ Patients with mental retardation or epilepsy, brain tumors, and psychoactive substance abuse.

After adjusting for the selection criteria, a total of 30 cases were recruited into the study ( 20 males and 10 females), with an average age of $34 \pm 12$ years. 17 cases were single and 13 were married, and the duration of education was $(12 \pm 4)$ years. The average age of onset was $(27 \pm 9)$ years, the time from onset of disease to first treatment was (12 \pm 3$)$ months, and the duration of illness was $(78 \pm 12)$ months.

Control group: The outpatients or inpatients diagnosed as NDS were evaluated by the SDS scale and the DS patients were excluded during the same period. In addition to the inclusion criteria $₫$ of the DS patients, other inclusion criteria and exclusion criteria are the same as the case group. There were 30 cases, including 21 males and 9 females, the average age was ( $35 \pm 11)$ years. 15 cases were single, 15 married, and the duration of education was $(13 \pm 3)$ years. The average age of onset was $(30 \pm 9)$ years, the

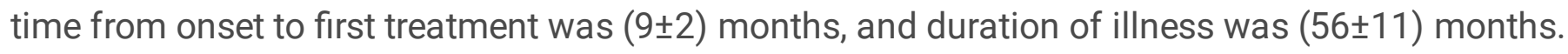


This study was approved by the Ethics Committee of Huai'an No. 3 People's Hospital of . Before the commencement of the study, the research purpose, method, and possible risks were explained to the subjects and their guardians. Also, written informed consent was obtained from the subjects and/or their guardians.

\section{Assessment}

\section{Assessment of socio-demographic and general information}

A self-designed questionnaire was used to collect the gender, age, education duration, marital status, and other sociodemographic characteristics such as the age of onset, duration of illness, time from onset of disease to first treatment, hospitalization frequency, name and dose of antipsychotics and use of anticholinergic drugs, and other general clinical data. The positive and negative syndrome scale (PANSS) was used to assess the current severity.

The dose of antipsychotics was converted to the equivalent dose of chlorpromazine for comparison. The specific conversion method is as follows: chlorpromazine $600 \mathrm{mg}=$ clozapine $600 \mathrm{mg}=$ risperidone $6 \mathrm{mg}=$ aripiprazole $30 \mathrm{mg}$ = olanzapine $20 \mathrm{mg}=$ quetiapine $750 \mathrm{mg}=$ sulpiride $1200 \mathrm{mg}=$ amisulpride $1200 \mathrm{mg}$. As the maximum therapeutic dose of each drug is considered to be the equivalent of the conversion dose, it was further converted according to the dose that was actually administered.

\section{Assessment of Deficit schizophrenia and non-defective schizophrenia}

The deficit syndrome (SDS) was conducted by the Chinese version of the schedule, which was compiled by Brian Kirkpatrick and translated into Chinese by Xiang Wang under the guidance of the original author. It consists of two parts. The first includes six aspects: emotion limitation, poor speech, narrow range of emotion, decreased interest, decreased purpose, and lack of social drive. The severity, primary and stability of negative symptoms were assessed. The severity was assessed on a scale of 5 from 0 to 4 , and the primary and stability of symptoms were scored according to answer "yes" or "no". The second part summarizes the results of the first part to evaluate whether the clinical manifestations of the patients meet the four aspects of the DS diagnostic criteria. This scale has been verified in the Chinese population and has good reliability and validity. Finally, patients with DS and NDS were classified according to the SDS scale.

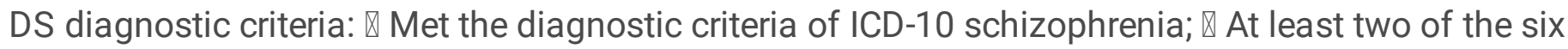

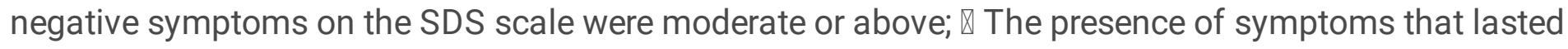
longer than a year and persisted even in clinical remission; $\mathbb{Q}$ It was the primary negative symptom, or negative symptoms secondary to psychiatric symptoms such as paranoia. Depression and anxiety were excluded, and negative symptoms secondary to extrapyramidal reactions, psychoactive substances, and mental retardation were also excluded.

\section{Assessment of white matter}


DTI data were collected using 1.5T magnetic resonance imaging (MRI) scanner(produced by the United Imaging company). Conventional brain scan sequences of the MRI included T1WI, T2WI, FLAIR T2WI, and DWI sequences, while the organic lesions of the whole brain were excluded. The DTI scan was performed using the spin-echo echo plane imaging (SE-EPI) sequence (TR=4600ms, TE $=106.4 \mathrm{~ms}$, FOV read at $230 \mathrm{~mm}$, FOV phase $220 \mathrm{~mm}$, read at 128 resolution, phase resolution 100 ). A total of 30 continuous layers were scanned with a slice thickness of $4.0 \mathrm{~mm}$, a diffusion sensitivity gradient direction of 24 , a diffusion sensitivity coefficient b of $1000 \mathrm{~s} / \mathrm{mm}^{2}$, and a scanning time of 5 mins $45 \mathrm{~s}$. Conventional MRI scan and DTI scan covered the whole brain.

For data processing, the tract-based spatial statistics (TBSS) method ${ }^{[7]}$ in the FSL (FMRIB Software Library, http://www.fmrib.ox.ac.uk/fsl) software package was used to analyze the DTI images of the white matter. Firstly, the eddy current correction was used to generate the whole brain mask before obtaining the individual FA figure. Then, the FA images of each subject were standardized to a spatial template of the standard brain atlas at the Montreal Neurological Institute (MNI) to make the average FA template and extract the tract skeleton. The white matter fibers of each of the subjects were registered to the tract skeleton, thus, completing the spatial normalization of the FA images. Finally, for each of the subjects in each group the average FA skeleton diagram (white matter skeleton diagram) calculated and the FA image was projected to the average white matter skeleton diagram for inter-group comparison.

\section{Statistical analysis}

SPSS 19.0 was used for statistical analysis. The measured data were expressed as $\mathrm{x} \pm \mathrm{s}$ and the independent sample t-test was used for comparison between the groups. The categorical variable was expressed by frequency and percentage, and the chi-square test was used for comparison. Spearman correlation analysis was used to compare the FA values in the white matter difference area between the two variables, and $p<0.05$ was considered to be statistically significant.

\section{Results}

There were no significant differences between the two groups in terms of gender, age, education time, marital status and other social demographic characteristics (P凶0.05). Comparison of clinical features: the age of onset of illness in the DS group was significantly lower than that in the NDS group. The duration from onset of disease to treatment and the duration of illness in the DS group were significantly longer than that of the NDS group, The administration of atypical antipsychotics was more, while the dose of the antipsychotics used (the equivalent dose of chlorpromazine) was lower, and the difference was statistically significant $(P<0.05)$. There was no significant difference in the use of anticholinergic between the two groups (P®0.05), See table.1.

\section{Table.1 Comparison of patient demographic data in two groups $(\mathrm{N}=60)$}




\begin{tabular}{|c|c|c|c|c|}
\hline Items & DS group $(n=30)$ & NDS group $(n=30)$ & $\mathrm{x}^{2} / \mathrm{t}$ & $p$ \\
\hline Gender male & 20 & 21 & 0.781 & 1.000 \\
\hline female & 10 & 9 & & \\
\hline Age (years) & $34 \pm 12$ & $35 \pm 11$ & 0.532 & 0.597 \\
\hline Education duration (year) & $12 \pm 4$ & $13 \pm 3$ & 0.644 & 0.527 \\
\hline Marital status (married) & 17 & 15 & 0.586 & 0.559 \\
\hline single & 13 & 15 & & \\
\hline Age of onset of disease (year) & $27 \pm 9$ & $30 \pm 9$ & 3.913 & 0.048 \\
\hline Time From onset to treatment (month) & $12 \pm 3$ & $9 \pm 2$ & 5.166 & $\varangle 0.001$ \\
\hline duration of illness (month) & $78 \pm 12$ & $56 \pm 11$ & 0.586 & 0.559 \\
\hline Antipsychotic atypical neuroleptics & 22 & 16 & 5.846 & 0.035 \\
\hline typical neuroleptics & 8 & 14 & & \\
\hline Equivalent dose of chlorpromazine (mg) & $211.09 \pm 35.33$ & $316.93 \pm 27.95$ & 5.285 & $\nabla 0.001$ \\
\hline Use of anticholinergic drugs yes & 18 & 21 & 1.931 & 0.384 \\
\hline No & 12 & 9 & & \\
\hline
\end{tabular}

The results of the TBSS analysis showed that the FA values in the right side of the knee of the corpus callosum (MNI:14,36,-7), the right anterior radio-coronal region ( $\mathrm{MIN}: 11,34,3)$, and the right hippocampal region $(\mathrm{MIN}: 30,34,16)$ in the DS patients were significantly lower compared with that of the NDS patients (Fig.1 and Table.2).

\section{Table.2 Comparison of the FA values of white matter changes between the two groups}

\begin{tabular}{lllll} 
Brain region & Voexl & $\mathrm{t}$ & $\mathrm{p}$ & $\mathrm{MNI}(\mathrm{x}, \mathrm{y}, \mathrm{z})$ \\
\hline The right knee of corpus callosum & 125 & -3.54 & 0.001 & $14,36,-7$ \\
\hline The right anterior radio-coronal region & 183 & -3.35 & 0.002 & $11,34,3$ \\
\hline The right hippocampal region & 110 & -3.474 & 0.002 & $30,34,16$
\end{tabular}

Note: the $t$ value is negative, indicating that FA values of the DS patients are lower than that of the NDS patients. 
Spearman correlation analysis was conducted between the above three brain regions with abnormal FA values in the DS group and the age, duration of illness, age of onset, time from onset to initial treatment, time of antipsychotic use, equivalent dose of chlorpromazine, and the score of PANSS scale. The results showed that the FA values in the right side of the knee of the corpus callosum (MNI:14,36,-7) was significantly correlated with the time from onset of disease to initial treatment and the PANSS-negative symptom score. In addition, the FA values in the right anterior radio-coronal region (MIN:11,34,3) was significantly correlated with PANSS negative symptoms, and the FA values in the right hippocampus region (MIN: 30,34,16) was significantly correlated with education duration , duration of antipsychotic use, and PANSS-negative symptoms. The results were statistically significant (p®0.05), See Table.3.

\section{Table.3 Correlation analysis of abnormal FA value in brain region with clinical variables in the DS group}

Brain region the right side of the knee of the corpus callosum

\section{The right anterior radio-coronal region}

The right

hippocampus region

\begin{tabular}{|c|c|c|c|}
\hline Age (years) & $r=-0.081, p=0.18$ & $r=-0.103, p=0.109$ & $r=-0.068 \rrbracket p=0.302$ \\
\hline Education year (years) & $r=-0.014, p=0.96$ & $r=-0.097, p=0.041$ & $r=-0.614, p=0.020 *$ \\
\hline Onset age (years) & $r=-0.049, p=0.461$ & $r=-0.052, p=0.413$ & $r=-0.07 \rrbracket p=0.28$ \\
\hline $\begin{array}{l}\text { Disease duration } \\
\text { (months) }\end{array}$ & $r=-0.047, p=0.423$ & $r=-0.034, p=0.601$ & $r=-0.03 \rrbracket p=0.58$ \\
\hline $\begin{array}{l}\text { Time from onset to } \\
\text { treatment (months) }\end{array}$ & $r=-0.350, p<0.001^{*}$ & $r=-0.336, p=0.562$ & $r=-0.02 \rrbracket p=0.72$ \\
\hline $\begin{array}{l}\text { Duration of } \\
\text { antipsychotic use } \\
\text { (months) }\end{array}$ & $r=-0.020, p=0.708$ & $r=-0.089, p=0.132$ & $\begin{array}{l}r=-0.140 \\
p=0.022^{\star}\end{array}$ \\
\hline $\begin{array}{l}\text { Equivalent dose of } \\
\text { chlorpromazine (mg) }\end{array}$ & $r=-0.018, p=0.714$ & $r=0.058, p=0.470$ & $r=-0.502 \rrbracket p=0.739$ \\
\hline $\begin{array}{l}\text { PANSS-positive } \\
\text { symptom score }\end{array}$ & $r=0.027, p=0.615$ & $r=-0.049, p=0.524$ & $r=-0.301 \rrbracket p=0.907$ \\
\hline $\begin{array}{l}\text { PANSS-negative } \\
\text { symptom score }\end{array}$ & $r=-0.157, p=0.007 *$ & $r=-0.306, p=0.048^{*}$ & $r=-0.637 \rrbracket p=0.040 *$ \\
\hline $\begin{array}{l}\text { PANSS general } \\
\text { pathological score }\end{array}$ & $r=0.053, p=0.408$ & $r=0.093, p=0.224$ & $r=-0.107 \rrbracket p=0.315$ \\
\hline
\end{tabular}

\section{Discussion}

FA values are the main quantitative index of DTI technology and represent the microstructure integrity of white matter fibers. A decrease in the FA suggests a reduction in glial cells, an impairment of the integrity of myelin sheaths, destruction of fiber microstructures, and damages to the intercortical loop connections ${ }^{[8]}$. In this study, the differences in free-water correction fraction anisotropy (FA) values of the 
white matter between the DS and NDS groups were investigated. The results showed that the FA values in the right side of the knee of the corpus callosum (MNl:14,36,-7), the right anterior radio-coronal region (MIN:11,34,3), and the right hippocampal region (MIN:30,34,16) in the DS patients were significantly lower than that of the NDS patients. Furthermore, there was a statistically significant correlation between the FA values of the three brain regions mentioned above and the duration of education, time from onset of disease to treatment, time of antipsychotic use, and the PANSS-negative symptoms score in the DS patients.

The corpus callosum is the largest connective fiber of the human brain, which is responsible for the transmission and integration of information in both hemispheres of the brain. The knee is located in front of the corpus callosum, and its white matter fibers connect to the bilateral prefrontal cortex. The destruction of its integrity will lead to damages in the bilateral prefrontal cortex connection, which is closely related to the function of the frontal lobe ${ }^{[9]}$. Previous studies have found subtle structural changes in the knees of the corpus callosum in schizophrenia that are thought to be caused by neurodysplasia ${ }^{[10]}$. In this study, the FA value on the right knee of the corpus callosum in the DS patients was significantly lower than that of NDS patients, suggesting that the damage of frontal lobe function-related brain area is more obvious in the DS patients. Meanwhile, it has been found that the FA value on the right knee of the corpus callosum is negatively correlated with the time from onset of disease to treatment and PANSSnegative symptoms, suggesting that the damage of the knee connection in the corpus callosum in the DS patients may be related to neuroplasticity-associated damages in the DS patients, besides the neurodysplasia.

The radio-crown refers to the radial white matter fibers from the inner capsule to the cerebral cortex, and the spatial arrangement of various projection fibers with different functions is currently not completely clear $^{[11]}$. Many studies have found varying degrees of damage to the right or bilateral radio-crown in patients with schizophrenia ${ }^{[12-13]}$. In this paper, the FA values in the right anterior radio-coronal region in patients with DS were lower compared with NDS patients, suggesting that the integrity of white matter fibers in the radio-crown is more seriously damaged. However, its mechanism is still unclear. In terms of the significant negative correlation between FA values in this brain region and PANSS-negative symptoms. It is likely that the neurophysiological basis of DS is not completely consistent with that of NDS.

The hippocampus is a part of the limbic system of the brain that is located in the medial temporal lobe and plays a role in short-term memory, long-term memory, and spatial positioning. It has structures such as the hippocampal horn and dentate gyrus, which act as a bridge between the body and the control center of the brain. The developed hippocampus indicates good memory while an impaired hippocampus results in varying degrees of cognitive impairment. Currently, studies on the hippocampus is one of the hotspots in the neurophysiological mechanism of schizophrenia ${ }^{[14]}$. In this study, it was found that the white matter fiber in the right hippocampal region was more seriously damaged in DS patients, and it was significantly correlated with duration of education, duration of antipsychotic use, and the PANSS- 
negative symptoms score. This supports the conclusions of some previous studies, providing a possible explanation for the more severe cognitive impairment seen in DS patients ${ }^{[15]}$.

Unilateral brain dysplasia is one of the etiological hypotheses of schizophrenia. It is believed that schizophrenia is caused by imbalanced development of the left and right cerebral hemispheres, and that individuals whose left hemisphere develops later than the right side in the embryo period have a higher risk of schizophrenia ${ }^{[16]}$. In this study, it was found that the three brain regions with differences in FA values between DS and NDS patients were all on the right side, and the mechanism may be related to the abnormal brain development. However, this needs to be further investigated .

The limitation of this study is that the two study groups are not homogeneous enough in terms of the patient demographic data. This might have affected the interpretation of the research results.

Furthermore, it is believed that the brain regions with different white matter damage are closely related to cognitive function. In future research, patients with better homogeneity will be selected. In addition, cognitive function assessment tools will be used to further investigate the damages caused by the structural integrity of white matter fibers in the three brain regions of DS so as to comprehensively and accurately explore the neurophysiological mechanisms of DS.

\section{Conclusion}

Schizophrenic patients with defective symptoms, the structural integrity of white matter fibers was more seriously damaged in the three brain regions including the right knee of the corpus callosum, the right anterior region of the right radiative crown, and the right hippocampus. These white matter lesions are closely related to patients characteristics such as duration of education, duration from onset of disease to treatment, duration of anti-psychotic use, and the severity of the negative symptoms.

\section{Abbreviations}

DS:Deficit schizophrenia;

NDS: non-defective schizophrenia;

PANSS: the positive and negative syndrome scale ;

DTI:Diffuse Tensor Imaging;

TBSS: the tract-based spatial statistics ;

FA:fractional anisotropy

\section{Declarations}

Ethics approval and consent to participate 
This study was approved by the Ethics Committee of Huai'an No.3 People's Hospital of . Before the commencement of the study, the research purpose, method, and possible risks were explained to the subjects and their guardians. Also, written informed consent was obtained from the subjects and/or their guardians.series analysis will be followed.

\section{Consent for publication}

Not Applicable.

\section{Availability of data and materials}

The datasets generated and/or analysed during the current study are not publicly available due to lack of ethics committee permission and not having been part of the consent process. The structure of the data set and the coding specification are available from the authors. Any other reasonable request will be raised with the regional ethics committee and health care provider.

\section{Competing interests}

The authors declare that they have no competing interests.

\section{Funding}

This study was supported by the Huai'an Key Research and Development Program (Social Development) under Grant No.HAB201733. The study design, results, conclusions, and opinions expressed in this paper are those of the authors and no endorsement by Huai'an goverment is intended or should be inferred.

\section{Authors' contributions}

WJS, TPS and CBH conceptualized the initial study design and developed the initial draft of the manuscript. LRZ, HF, PY, ZW and CJW extracted the clinical data. All authors interpreted the data, provided constructive feedback during manuscript development, and read and approved the final manuscript.

\section{Acknowledgements}

We are grateful to Dr.Jian-nan Zhu and Yun-shan Zhou for providing valuable data and the patient's relatives' agreement for publication.

\section{Corresponding author}

Correspondence to Cheng-bing Huang.

\section{References}


1. Takahashi T, Takayanagi Y, Nishikawa Y, et al. Brain neurodevelopmental markers related to the deficit subtype of schizophrenia[J]. Psychiatry Res Neuroimaging. 2017;266:10-18. doi: 10.1016/j.pscychresns.2017.05.007.

2. Yu M, Tang X, Wang X, et al. Neurocognitive Impairments in Deficit and Non-Deficit Schizophrenia and Their Relationships with Symptom Dimensions and Other Clinical Variables[J]. PLoS One. 2015;10(9):e0138357. doi: 10.1371/journal.pone.0138357. eCollection 2015.

3. Ohtani T, Bouix S, Hosokawa T, et al. Abnormalities in white matter connections between orbitofrontal cortex and anterior cingulate cortex and their associations with negative symptoms in schizophrenia: a DTI study [J]. Schizophr Res. 2014;157(1-3):190-7. doi:

10.1016/j.schres.2014.05.016.

4. Yang Fan, Li Peikai, Hou Yu, et al. The abnormality of white matter and correlation with clinical symptoms in chronic schizophrenia [J]. Chinese Journal of Psychiatry, 2014,47(1):2630.DOI:10.3760/cma.j.issn.1006-7884.2014.01.007.

5. Ohtani T, Bouix S, Hosokawa T, et al. Abnormalities in white matter connections between orbitofrontal cortex and anterior cingulate cortex and their associations with negative symptoms in schizophrenia: a DTI study[J]. Schizophr Res. 2014 Aug;157(1-3):190-7. doi: 10.1016/j.schres. 2014. 05.016.

6. Wang Xiang, Yao Shuqiao, Fan Xuhui, et al. The Chinese Version of the Schedule for the Deficit Syndrome: Reliability and Validity [J]. Chinese Journal of Clinical Psychology, 2005,13(4):392395.DOI:10.3969/j.issn.1005-3611.2005.04.005.

7. Wang Xingrui, Xi Yibin, Zhu Yuanqiang, et al. White matter microstructure in first-episode schizophrenia: diffusion tensor imaging study using tract-based spatial statistics analysis [J]. Journal of Wuhan University (Medical edition), 2019,40(3):414-419.DOI冈14188/j.16718852.2019.0018.

8. Zhao Ke, Wang Qiang, Deng Wei, et al. The role of the white matter integrity of the anterior commissure in cognitive functions in first-episode schizophrenia [J]. Chinese Journal of Neuropsychiatric Disorders, 2014,(1):16-20. DOI:10.3936/j.issn.1002-0152.2014.01.004.

9. Roland JL, Snyder AZ, Hacker CD, et al. On the role of the corpus callosum in interhemispheric functional connectivity in humans[J]. Proc Natl Acad Sci USA. 2017;114(50): 13278-13283. doi: 10.1073/ pnas. 1707050114 .

10. Shahab S, Stefanik L, Foussias G, et al. Sex and Diffusion Tensor Imaging of White Matter in Schizophrenia: A Systematic Review Plus Meta-analysis of the Corpus Callosum[J]. Schizophr Bull. 2018;44(1):203-221. doi: 10.1093/schbul/sbx049.

11. Koshiyama D, Fukunaga $\mathrm{M}$, Okada $\mathrm{N}$,et al. Role of frontal white matter and corpus callosum on social function in schizophrenia[J]. Schizophr Res. 2018 Dec;202:180-187. doi: 10.1016/j.schres.2018.07.009.

12. Kelly $\mathrm{S}$, Jahanshad N, Zalesky A, et al.Widespread white matter microstructural differences in schizophrenia across 4322 individuals: results from the ENIGMA Schizophrenia DTI Working 
Group[J]. Mol Psychiatry. 2018;23(5):1261-1269. doi: 10.1038/mp.2017.170.

13. Subramaniam K, Gill J, Fisher M,et al. White matter microstructure predicts cognitive training-induced improvements in attention and executive functioning in schizophrenia[J]. Schizophr Res. 2018 Mar;193:276-283. doi: 10.1016/j.schres.2017.06.062.

14. Nakahara S, Matsumoto M, van Erp TGM.Hippocampal subregion abnormalities in schizophrenia: A systematic review of structural and physiological imaging studies[J]. Neuropsychopharmacol Rep. 2018;38(4):156-166. doi: 10.1002/npr2.12031.

15. Bähner F1, Meyer-Lindenberg A.Hippocampal-prefrontal connectivity as a translational phenotype for schizophrenia[J]. Eur Neuropsychopharmacol. 2017;27(2):93-106. doi:

10.1016/j.euroneuro.2016.12.007.

16. Wu Daxing, Yao Shuqiao, Cheng Zaohuo, e tal. Study of 32 Channel t-test Significance Probability Mapping of Visual P100 in Schizophrenics [J]. Chinese Journal of Clinical Psychology, 2001,9(4):250-252.DOI:10.3969/j.issn.1005-3611.2001.04.004.

\section{Figures}

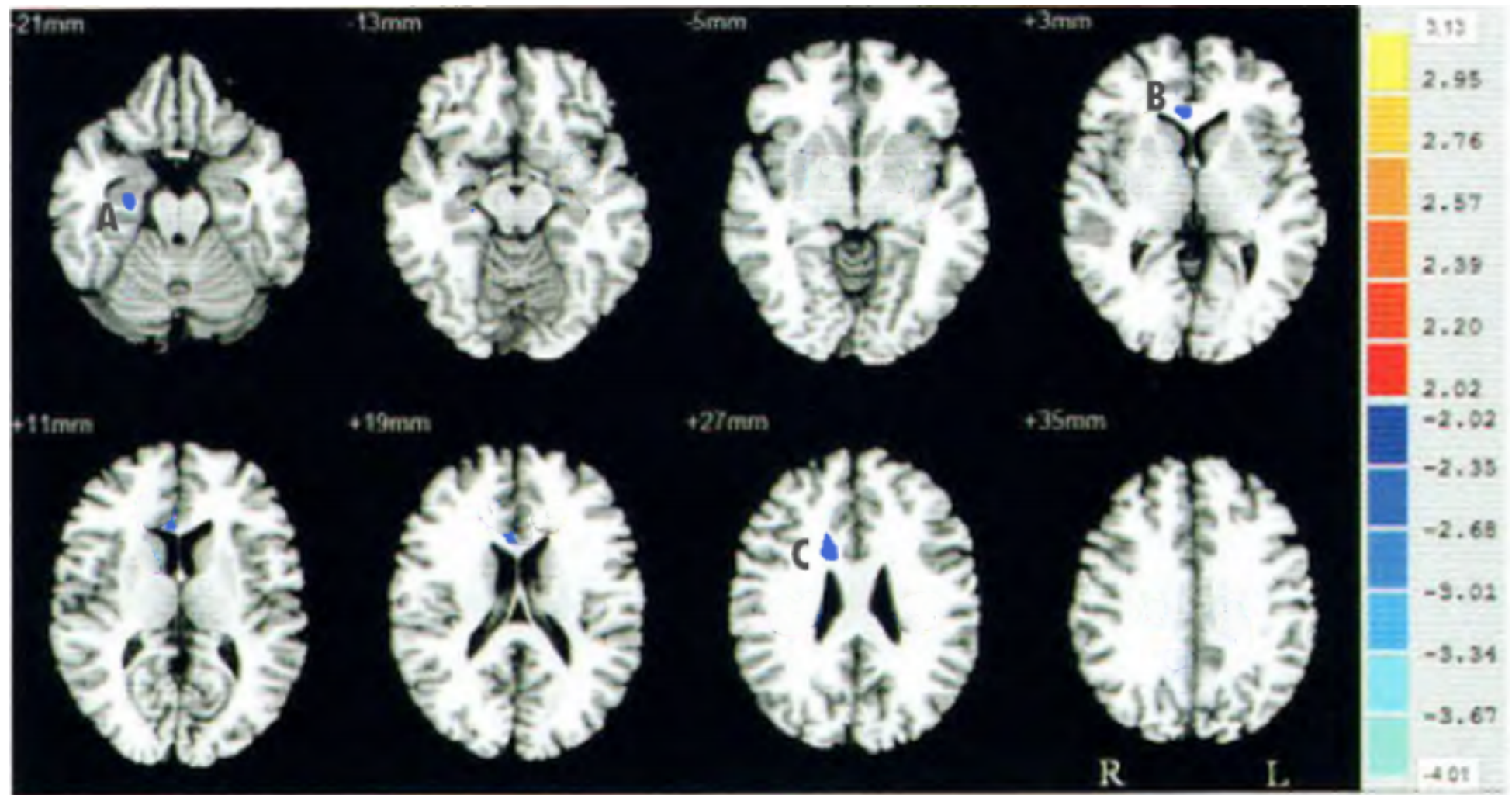

\section{Figure 1}

Comparison of the white matter changes between the two groups. A: the right hippocampal region; B: the right knee of corpus callosum; $\mathrm{C}$ : the right anterior radio-coronal region 